\title{
MODELO EXPERIMENTAL DE INDUÇÃO DE LESÃO OXIDATIVA HEPÁTICA EM RATOS POR HALOTANO
}

\author{
Luis Josino BRASIL ${ }^{1}$, José Luiz Gomes do AMARAL², Claudio Galeano ZETTLER ${ }^{3}$, \\ Claudio Augusto MARRONI ${ }^{4}$, Rafael VERCELINO ${ }^{5}$ e Norma MARRONI ${ }^{5}$
}

\begin{abstract}
RESUMO - Racional - O anestésico halotano pode ser metabolizado redutivamente a intermediários reativos que podem iniciar a lipoperoxidação acompanhada de injúria hepática. O tratamento prévio com hipóxia e fenobarbital em ratos aumenta o metabolismo do halotano e o estresse oxidativo e causa mudanças nas enzimas antioxidantes no fígado com dano hepático. Métodos - Investigou-se o efeito do halotano na lipoperoxidação e histologia hepáticas após o aumento do metabolismo redutor do halotano induzido pela hipóxia e fenobarbital. Vinte e cinco ratos machos Wistar foram divididos em cinco grupos: Co (controle), $\mathrm{HO}_{14}$ (Halotano/Hipóxia), F (Fenobarbital), $\mathrm{O}_{14}$ (Hipóxia) e H (Halotano). Após 24 horas os ratos foram sacrificados, seus fígados foram retirados para determinar quimiluminescência, substâncias que reagem ao ácido tiobarbitúrico, enzimas antioxidantes, superóxido dismutase, catalase e amostras de sangue foram tomadas para determinar AST e a ALT. A avaliação histopatológica foi realizada pela técnica de hematoxilina-eosina. Os dados da avaliação histológica foram apresentados através de mediana e amplitude entre quartis. Resultados e Conclusões - A exposição ao halotano/hipóxia causou lipoperoxidação hepática e mudanças significativas na atividade das enzimas antioxidantes. Além disso, provocou lesão histopatológica do fígado e aumento significativo dos níveis plasmáticos de AST e ALT.
\end{abstract}

DESCRITORES - Halotano. Hepatotoxicidade. Estresse Oxidativo. Hipoxia. Antioxidante.

\section{INTRODUÇÃO}

Muitos pesquisadores têm dedicado esforços para elucidar os mecanismos subjacentes à lesão hepática provocada pelo anestésico halotano. Demonstrou-se a formação de espécies reativas de oxigênio in vivo após a exposição ao halotano pela espectroscopia paramagnética eletrônica ${ }^{(9)}$ ou pela quantificação de fluorino sub 2-isoprostanos ${ }^{(1)}$. No entanto, a formação de radicais derivados de lipídios tem sido controversa ${ }^{(10)}$ ou não observada ${ }^{(18)}$. Vários estudos têm indicado que a hipóxia aumenta a ligação de metabólitos do halotano a componentes da fração microssomal hepática ${ }^{(16)}$.

Em um estudo, ratos com indução enzimática prévia, foram expostos por 2 horas, a concentrações de $\mathrm{O}_{2}$ de $14 \%$ e halotano $1 \%$, resultando com necrose centro-lobular em $24 \mathrm{~h}$ (fenobarbital $1 \mathrm{mg} / \mathrm{mL}$ na água de beber), por 10 dias antes do evento ${ }^{(14)}$. Esses resultados suportam a hipótese de que o halotano é metabolizado até intermediários hepatotóxicos em um modelo associado ao citocromo p-450 redutivo ou não-dependente de $\mathrm{O}_{2}$. LIND et al. ${ }^{(11)}$ demonstraram que o grau de hepatotoxicidade máxima determinada por alterações morfológicas e atividade de
ALT correlaciona-se com a concentração de halotano e de citocromo P-450 hepático.

Existem vários modelos animais que reproduzem lesão oxidativa hepática, a maioria possui alta mortalidade e tempo gasto para seu êxito em relação ao modelo com halotano. Dentre os agentes etiológicos utilizados destacam-se o álcool, o tetracloreto de carbono e a ligadura de canal biliar ${ }^{(5,13,19)}$.

O objetivo do presente trabalho foi confirmar se o halotano causa peroxidação lipídica e alteração nas enzimas antioxidantes in vivo em curto período de tempo e pode, portanto, ser utilizado como modelo experimental de lesão oxidativa hepática. Determinaram-se os efeitos do fenobarbital, da hipóxia e do halotano separadamente nesse processo. Finalmente, determinou-se se a peroxidação lipídica é acompanhada de injúria hepática (alterações estruturais e de aminotransferases hepáticas).

\section{MÉTODOS}

Foram utilizados ratos machos, Wistar, com peso entre 250 e $350 \mathrm{~g}$, provenientes do Biotério do Instituto

\footnotetext{
${ }^{1}$ Serviço de Anestesiologia, Irmandade Santa Casa de Misericórdia de Porto Alegre. RS; ${ }^{2}$ Departamento de Anestesiologia e Cuidados Intensivos, Universidade Federal de São Paulo, SP; ${ }^{3}$ Departamento de Patologia, Fundação Faculdade Federal de Ciências Médicas de Porto Alegre, RS; ${ }^{4}$ Departamento de Hepatologia, Fundação Faculdade Federal de Ciências Médicas de Porto Alegre, RS; ${ }^{5}$ Laboratório de Fisiologia Experimental, Hospital das Clínicas de Porto Alegre, Universidade Federal do Rio Grande do Sul, Porto Alegre, RS Correspondência: Dr. Luis Josino Brasil - Av. Guaporé, 530 - Petrópolis - 90470-230 - Porto Alegre, RS. E-mail: ljbrasil@yahoo.es
} 
de Ciências Básicas da Saúde da Universidade Federal do Rio Grande do Sul, Porto Alegre, RS.

Todos os procedimentos realizados estavam de acordo com as normas estabelecidas pela comissão de pesquisa e ética em saúde contidas na "Pesquisa em Saúde e Direito dos Animais", de autoria do grupo de pesquisa e pós-graduação do Hospital de Clínicas de Porto Alegre ${ }^{(6)}$.

Os animais foram divididos em cinco grupos experimentais:

Grupo $\mathrm{CO}$ - sem tratamento; grupo $\mathrm{HO}_{14}$ - receberam fenobarbital na água de beber ad libitum por 10 dias e foram submetidos a concentração hipóxica de $\mathrm{O}$ a $14 \%$ e halotano a $1 \%$ por $2 \mathrm{Hs}$; grupo $\mathrm{F}$ - receberam fenobarbital na água de beber ad libitum por 10 dias; grupo $\mathrm{O}_{14}$ - foram submetidos a concentração hipóxica de $\mathrm{O}_{2}$ a $14 \%$ por $2 \mathrm{Hs}$; grupo $\mathrm{H}$ - foram submetidos a concentração de halotano a $1 \%$ por $2 \mathrm{~h}$.

Os animais foram inalados no interior de uma caixa padronizada $^{(13)}$ tiveram a concentração de oxigênio aferida através de um oxímetro ambiental e foram monitorados por uma câmera na caixa inalatória. Para obtenção dos tecidos, os animais dos grupos inalados $\left(\mathrm{O}_{14} \mathrm{H}\right.$, e $\left.\mathrm{HO}_{14}\right)$ foram sacrificados 24 horas após a exposição, junto com os demais grupos através de laparotomia mediana e amostras de tecido hepático e sangue foram colhidas. Os tecidos foram colocados em solução de tampão fosfato $20 \mathrm{mM}$, ph 7,4 (KCl $140 \mathrm{mM})$ na proporção de $9 \mathrm{~mL}$ por grama de tecido. Os fígados foram homogeneizados por 40 segundos, a temperatura de $0-2^{\circ}$ centígrados. Os homogeneizados foram centrifugados por 10 minutos a $4000 \mathrm{rpm}$. Desprezou-se o precipitado de cada amostra e o sobrenadante foi armazenado e congelado a $-80^{\circ}$ centígrados $^{(14)}$.

Para a avaliação da lipoperoxidação utilizou-se a técnica de quimiluminescência (QL) e das substâncias que reagem ao ácido tiobarbitúrico (TBARS).

O método para determinar a QL consistiu em adicionar um hidroperóxido orgânico de origem sintética (hidroperóxido de tert-butila) ao homogeneizado de tecido em estudo. A QL foi medida em um contador com o circuito de coincidência desconectado e utilizado o canal de tritio operando como um luminômetro. Os resultados foram expressos em contagem por segundo (cps) por mg de proteína ${ }^{(15)}$.

A técnica de TBARS foi realizada conforme descrito anteriormente $^{(16)}$ utilizando $1,5 \mathrm{~mL}$ de solução de ácido tricloroacético (TCA) $10 \%, 0,5 \mathrm{~mL}$ de homogeneizado de tecido hepático, 1,0 mL de ácido tiobarbitúrico (TBA) 67\% e água destilada. Essa mistura foi aquecida a $100^{\circ} \mathrm{C}$ em banho-maria durante 15 minutos e resfriada em gelo. Após adição de $3 \mathrm{~mL}$ de álcool n-butílico e agitação por 40 segundos, esse material foi centrifugado por 10 minutos a $3000 \mathrm{rpm}$ e obteve-se um sobrenadante corado, resultante da reação de malondialdeído e outros subprodutos liberados na lipoperoxidação. O sobrenadante foi colocado em cubeta de quartzo para leitura a $535 \mathrm{~nm}$ em espectofotômetro. Os resultados foram expressos em nanomoles por miligramas de proteína (nmoles/mg prot).

A determinação da atividade da enzima superóxido-dismutase (SOD) foi baseada na inibição da reação do radical superóxido com a adrenalina. A SOD, presente na amostra em estudo, competiu pelo radical superóxido através do sistema de detecção. Os resultados foram expressos em unidades de SOD por mg de proteína ${ }^{(17)}$.

A atividade da enzima catalase (CAT) foi realizada conforme modelo previamente descrito ${ }^{(11)}$, baseado na velocidade de consumo de peróxido de hidrogênio na amostra de tecido hepático. Utilizaram-se, nessa técnica, $955 \mu \mathrm{l}$ de tampão fosfato $(50 \mathrm{nM}$, ph 7,4), e $10 \mu \mathrm{l}$ de homogeneizado de tecido hepático em cubeta de quartzo, onde foi adicionado $35 \mu \mathrm{l}$ de peróxido de hidrogênio (0,3 M). A velocidade da decomposição do $\mathrm{H}_{2} \mathrm{O}_{2}$ foi medida espectofotometricamente a $240 \mathrm{~nm}$. Os resultados foram expressos em $\mathrm{nmol} / \mathrm{mg}$ de proteína.

Pela técnica da punção do plexo venoso retro-orbital com tubo de capilar de vidro heparinizado, colheu-se sangue utilizado para as dosagens de AST e ALT e. Esta dosagens foram realizadas no Laboratório Central da Santa Casa de Misericórdia de Porto Alegre.

Cortes com $5 \mu \mathrm{m}$ de fixado, com tecidos hepáticos fixados em formalina e embebidos em parafina foram submetidos a coloração com hematoxilina e eosina e analisadas em diferentes aumentos (100 a 400X). Os achados foram classificados de acordo com os critérios clássicos para lesão hepática por halotano descritos anteriormente ${ }^{(6)}$.

\section{1 - Normal}

2 - Alguma pequena variação ou ocasional vacuolização/células em balão

3 -Ocasional necrose centro lobular

4 -Muitas áreas de necrose centro lobular

5 -Necroses centro lobulares confluentes

A análise estatística foi feita por ANOVA, seguido de teste de TUKEY como pós-teste, sendo considerados significativos os resultados $\operatorname{com} P$ menores que 0,01 . Os resultados foram expressos como média \pm desvio padrão. Os dados da avaliação histológica foram apresentados através de mediana e amplitude entre quartis.

\section{RESULTADOS}

\section{Lipoperoxidação hepática}

Nota-se que a lipoperoxidação dos animais que receberam o modelo completo de lesão hepática por halotano $\left(\mathrm{HO}_{14}\right)$ aumentou significativamente, quando comparada ao grupo controle, tanto na técnica de QL quanto por TBARS $(P<0,01)$. Observa-se também que os animais que receberam o modelo incompleto $(\mathrm{F}$, $\mathrm{O}_{14}$ e H) não apresentaram lipoperoxidação significativamente superior ao grupo controle $(P>0,01)$.

TABELA 1. Avaliação da lipoperoxidação pela quimiluminescência (QL)

\begin{tabular}{lc}
\hline & $\begin{array}{c}\text { QL } \\
\text { (cps/mg proteína) }\end{array}$ \\
\hline Grupo $\mathrm{CO}$ & $3218,71 \pm 424,42$ \\
Grupo $\mathrm{HO}_{14}$ & $4499,46 \pm 307,45 *$ \\
Grupo F & $3929,97 \pm 260,42$ \\
Grupo $\mathrm{O}_{14}$ & $2868,83 \pm 862,58$ \\
Grupo H & $4273,34 \pm 492,73$ \\
\hline
\end{tabular}

Avaliação da QL nos diferentes modelos experimentais. Os resultados estão expressos em média \pm desvio padrão da média.

*Diferença significativa em relação ao grupo controle $(P<0,0)$ 
TABELA 2. Avaliação da lipoperoxidação pelo método de substancias que reagem ao ácido tiobarbitúrico (TBARS)

\begin{tabular}{lc}
\hline & $\begin{array}{c}\text { TBARS } \\
\text { (nmoles/mg proteína) }\end{array}$ \\
\hline Grupo $\mathrm{CO}$ & $0,25 \pm 0,02$ \\
Grupo $\mathrm{HO}_{14}$ & $0,41 \pm 0,05^{*}$ \\
Grupo $\mathrm{F}$ & $0,36 \pm 0,07$ \\
Grupo $\mathrm{O}_{14}$ & $0,35 \pm 0,07$ \\
Grupo $\mathrm{H}$ & $0,32 \pm 0,06$ \\
\hline
\end{tabular}

Avaliação de TBA-RS nos diferentes modelos experimentais. Os resultados estão expressos em médoa \pm desvio padrão da média.

Diferença significativa em relação ao grupo controle $(P<0,01)$

\section{Enzimas antioxidantes}

Houve aumento significativo na atividade da enzima superóxidodismutase (SOD) no grupo $\mathrm{HO}_{14}$ em relação ao grupo controle, conforme mostra a Figura 1, e não foram observadas diferenças nos demais grupos em relação ao grupo controle. Observou-se redução significativa na atividade da enzima catalase (CAT) nos grupos $\mathrm{HO}_{14}, \mathrm{H} \mathrm{e} \mathrm{O}_{14}$ em relação ao controle enquanto o grupo $\mathrm{F}$ foi semelhante ao controle, conforme mostra o Figura 2.

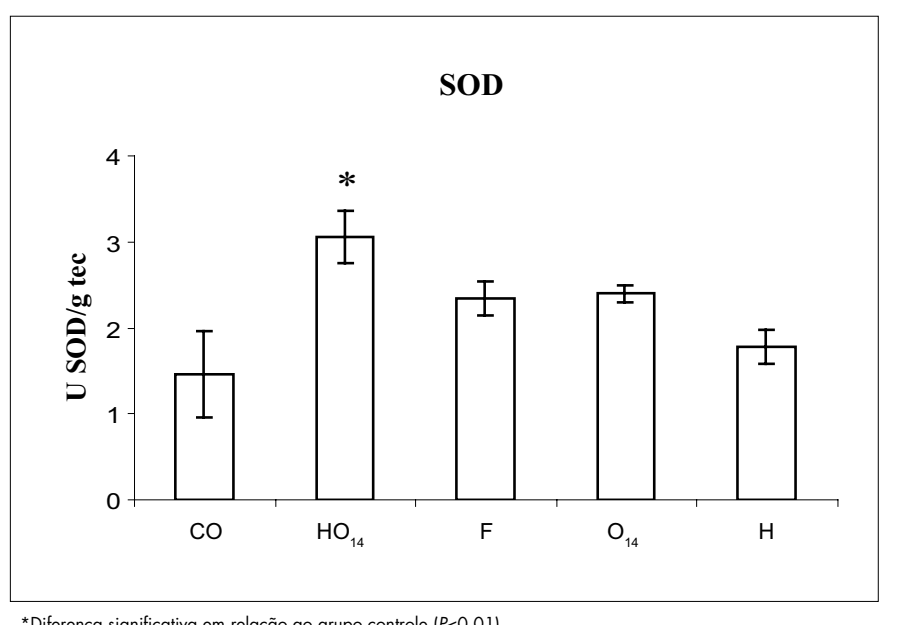

FIGURA 1. Valores da atividade da enzima superóxido dismutase (SOD)

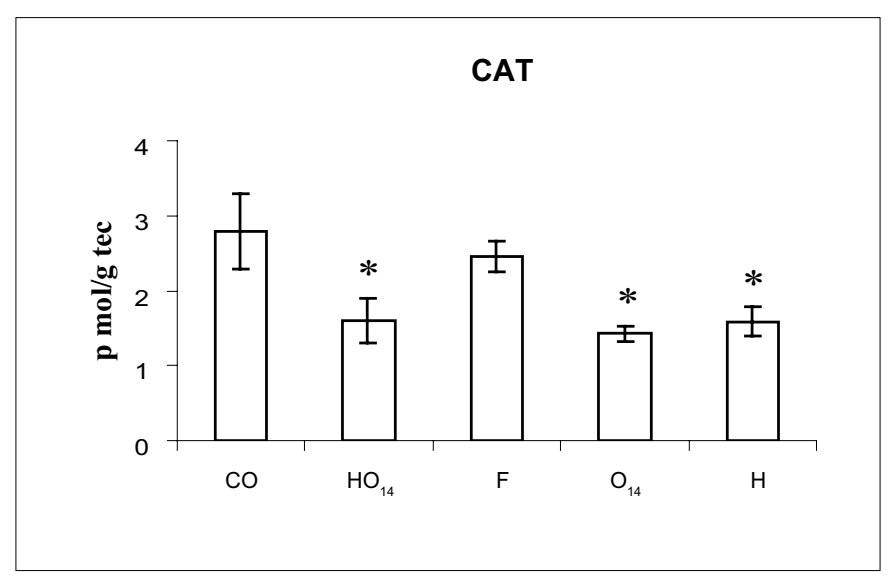

* Diferença significativa em relação ao grupo controle $(P<0,01)$

FIGURA 2. Valores da atividade da enzima catalase (CAT)

\section{Enzimas hepáticas}

Houve aumento significativo na atividade da enzima AST e ALT no grupo $\mathrm{HO}_{14}$ em relação ao grupo controle, sendo os demais grupos sem diferença significativa em relação ao controle.

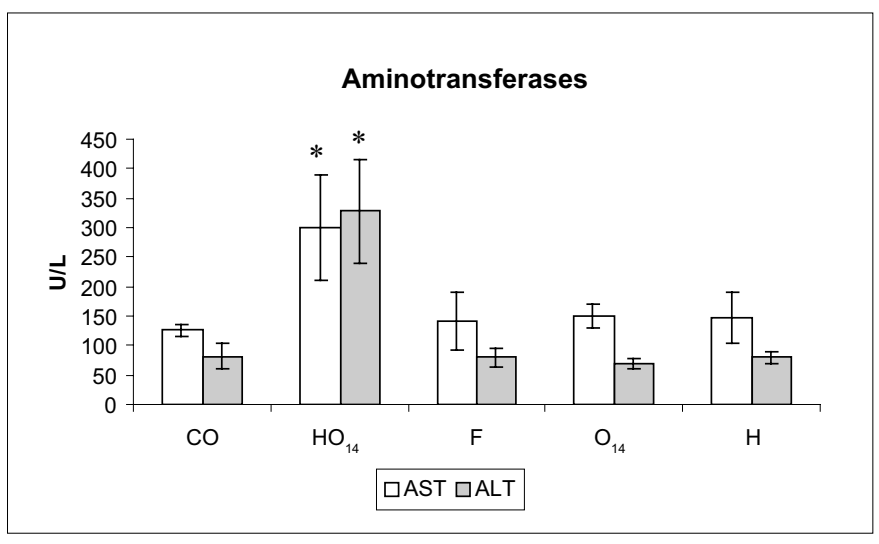

* Diferença significativa em relação ao grupo controle $(P<0,01)$

FIGURA 3. Valores de aspartato aminotransferase (AST) e alanino aminotrasferase (ALT) dos diferentes grupos.

\section{Análise histológica}

Os índices histopatológicos da extensão da injúria halotanoinduzida foram maiores que $3 \mathrm{em}$ todos os animais do grupo $\mathrm{HO}_{14}$ e menor que 3 em todos os animais dos demais grupos (Tabela 3). Fotos representativas dos escores $4\left(\mathrm{HO}_{14}\right)$ e 1 (histologia normal-grupo controle) são apresentadas nas Figuras 4 e 5.

TABELA 3. Escore de lesão hepática entre os diferentes grupos

\begin{tabular}{lccccc}
\hline Variáveis & $\mathrm{CO}$ & $\mathrm{HO}_{14}$ & $\mathbf{F}$ & $\mathrm{O}_{14}$ & $\mathbf{H}$ \\
\hline $\mathrm{n}$ & 5 & 5 & 5 & 5 & 5 \\
média & 1,00 & 4,00 & 1,00 & 1,00 & 1,00 \\
Percentil $25^{0}-75^{\circ}$ & 0,5 & 0,5 & 0,5 & 0,5 & 0,5 \\
\hline Injúria hepática: & & & & & \\
Médio $(\geq 0)$ & $(100 \%)$ & $(0 \%)$ & $(80 \%)$ & $(80 \%)$ & $(80 \%)$ \\
Moderado $(\geq 2)$ & $(0 \%)$ & $(0 \%)$ & $(20 \%)$ & $(20 \%)$ & $(20 \%)$ \\
Extenso $(\geq 3)$ & $(0 \%)$ & $(100 \%)$ & $(0 \%)$ & $(0 \%)$ & $(0 \%)$ \\
\hline
\end{tabular}

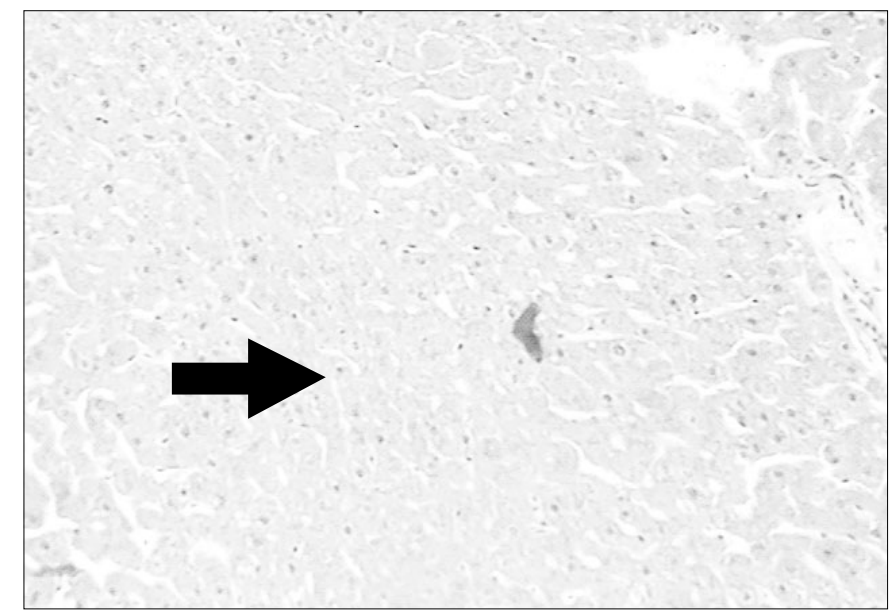

FIGURA 4. Histologia do fígado de um rato do grupo controle (CO), mostrando as estruturas hepáticas normais. (H\&E 40X) 


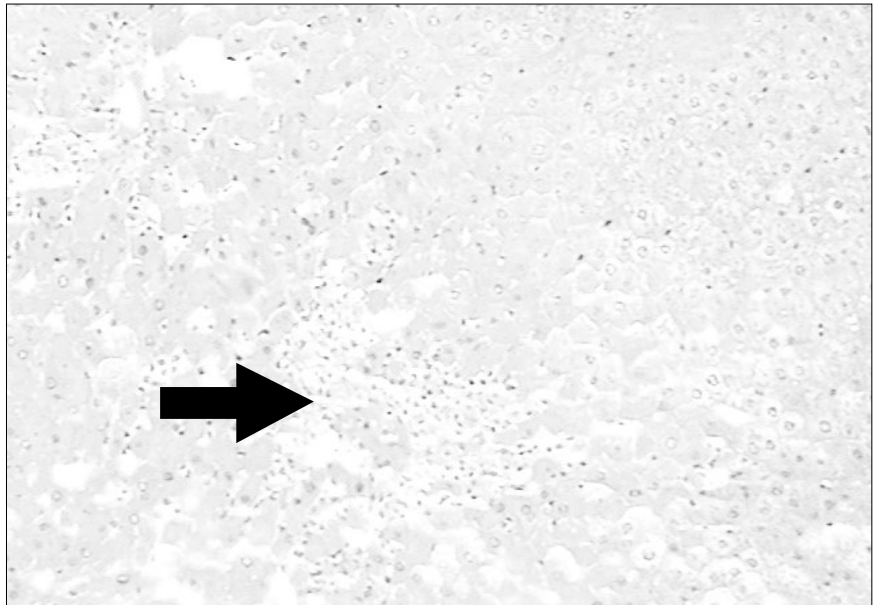

FIGURA 5. Histologia do fígado de um rato do grupo $\mathrm{HO}_{14}$, mostrando inflamação e necrose centro lobular. (H\&E 40X)

\section{DISCUSSÃO}

Procurou-se, inicialmente, reproduzir um modelo experimental de lesão hepática por halotano a partir do modelo clássico associado à hipóxia ${ }^{(6)}$. Durante a reprodução não houve qualquer perda de animais, adicionalmente o modelo necessitou de apenas 10 dias para ser completado. Esses dois aspectos representam vantagem em relação a outros modelos animais de lesão oxidativa hepática ${ }^{(8,9,10)}$.

Os resultados obtidos na avaliação da peroxidação lipídica e enzimas antioxidantes evidenciaram aumento do estresse oxidativo como previamente demonstrado ${ }^{(2,3)}$, associados à modificação das enzimas antioxidantes nos animais do grupo $\mathrm{HO}_{14}$. Esses achados sugerem uma relação entre as alterações encontradas nos marcadores de injúria hepática (aminotransferases e histopatologia) provocados pelo modelo completo e a formação de espécies reativas de oxigênio pelo sistema microssomal hepático.

A avaliação da peroxidação lipídica, demonstrou a impossibilidade da hipóxia, fenobarbital ou halotano isoladamente provocarem resposta de indução na peroxidação lipídica em relação ao controle para o nível de significância estabelecido $(P<0,01)$. Isso reforça a hipótese de que o mecanismo envolvido na lesão oxidativa hepática por halotano é mais intenso com indução enzimática prévia, baixas concentrações de $\mathrm{O}_{2}$ e exposição ao halotano aplicados simultaneamente ${ }^{(2)}$. A resposta significativa de consumo da enzima CAT nos grupos $\mathrm{He}_{14}$ sugerem mecanismos adaptativos das defesas antioxidantes que podem ter contribuído para impedir o aumento significativo na peroxidação hepática e nas aminotransferases bem como contribuído para a ausência de alterações histopatológicas nesses grupos.

Observou-se consumo significativo da enzima CAT no grupo $\mathrm{HO}_{14}$ associado a aumento significativo da $\mathrm{SOD}$, sendo que o responsável pelo efeito na SOD poderia ser o aumento na produção do ânion superóxido, potente radical livre, que teria provocado aumento adaptativo na SOD. O mecanismo proposto é que o ânion superóxido transformado em peróxido de hidrogênio forma o radical hidroxil, principal iniciador da lipoperoxidação ${ }^{(18)}$. Isso causa a remoção de um átomo de hidrogênio do ácido graxo polinsaturado presente na membrana celular resultando em lipoperoxidação. No grupo $\mathrm{HO}_{14}$ apesar dos mecanismos adaptativos antioxidantes de consumo na CAT e aumento na SOD, confirmamos o aumento significativo.da peroxidação lipídica em relação ao controle.

As enzimas aminotransferases, quando elevadas, sugerem disfunção hepática, relacionada às lesões de destruição de tecidos ou alteração da permeabilidade celular ${ }^{(19)}$. Não se encontrou aumento significativo nas aminotransferases associado à importante alteração histopatológica nos fígados dos animais que receberam o modelo em relação ao grupo controle.

\section{CONCLUSÕES}

Pelos resultados obtidos, observou-se a ocorrência de lesão oxidativa hepática, acompanhada de alterações histopatológicas e enzimáticas nos animais submetidos ao agente tóxico, em tempo bastante curto comparativamente a outros modelos animais, o que permite o desenvolvimento de pesquisas que envolvam o estresse oxidativo hepático e serve de subsídio para futuras avaliações da potencial modificação do estado red-ox hepático por outros agentes. A utilização do halotano apresenta ainda a vantagem de sua fácil aquisição no mercado por ser uma droga utilizada clinicamente, ao contrário de outros modelos que necessitam de drogas de difícil obtenção devido à alta toxicidade (p.ex. $\mathrm{CCl}_{4}$ ). Adicionalmente verificou-se que os animais submetidos isoladamente ao fenobarbital, hipóxia e halotano, não apresentaram peroxidação lipídica e injúria hepática no nível de significância estabelecido. 
Brasil LJ, Amaral JLG, Zettler CG, Marroni CA, Vercelino R, Marroni CA. Experimental model of liver oxidative damage induction in rats by halothane. Arq Gastroenterol. 2007;4(1):73-7.

ABSTRACT - Background - The anesthetic halothane can be reductively metabolized to reactives intermediates that may initiate lipid peroxidation accompanied by hepatic injury. Hypoxia and phenobarbital pretreatment in rats increases metabolism of halothane, the oxidative stress, cause liver antioxidant enzymes changes and tissue damage. Aims - We investigated the effect of halothane on hepatic lipid peroxidation and on hepatic histology after increases reductive metabolism of halothane caused by hypoxia and phenobarbital pretreatment. Methods - Twenty-five male wistar rats were divided in five equals groups: $\mathrm{CO}$ (Control), $\mathrm{HO}_{14}$ (Halothane/Hypoxia), $\mathrm{F}$ (fenobarbital alone), $\mathrm{O}_{14}$ (Hypoxia alone) and $\mathrm{H}$ (Halothane alone). After 24 hours the rats were killed, their livers removed to determine chemoluminescence, thiobarbituric acid-reactive substances, catalase, superoxide dismutase, and blood samples were taken to determine AST and ALT. The histopathologic evaluation was performed with hematoxylin and eosin staining. Histopathologic scores are presented as 25th-75th percentile/range values and median \pm range. Results/Conclusion - Halothane-hypoxic exposure resulted in a significant changes in the activities of antioxidant enzymes, and induced hepatic lipoperoxidation. Moreover it resulted in histopathologic liver injury as well as significant increase of serum activity of AST and ALT.

HEADINGS - Halothane. Hepatotoxicity. Oxidative stress. Hypoxia. Antioxidant.

\section{REFERÊNCIAS}

1. Awad JA, Horn JL, Roberts LJ 2nd, Franks JJ. Demonstration of halothane-induced hepatic lipid peroxidation in rats by quantification of F2-isoprostanes. Anesthesiology. 1996;84:910-6.

2. Boveris A, Chance B. The mitocondrial generation of hydrogen peroxide: general properties and effect of hyperbaric oxygen. Biochem J. 1973;134:707-16.

3. Brandão ABM, Marroni CA Testes de função hepática. In: Mattos A, Dantas W. Compêndio de hepatologia. 2.ed. São Paulo: Fundo Editorial BYK; 2001.

4. Buege JA, Aust SD. Microsomal lipid peroxidation. Meth Enzimol. 1978;52:302-9.

5. Cremonese RV, Pereira-Filho AA, Magalhães R, Mattos A, CG Zetler, Marroni CA, NAP Marroni. Cirrose experimental induzida pela inalação de CCl4 - adaptação da técnica e avaliação de peroxidação lipídica. Arq Gastroenterol. 2000;38:143-56.

6. Goldim JR, Raymundo MM. Pesquisa em saúde e direitos dos animais. 2.ed. Porto Alegre: HCPA; 1997.

7. Gonzalez-Flecha B, Llesuy S, Boveris A. Hydroperoxide-iniciated chemiluminescence: an assay for oxydative stress in biopses of liver, heart and muscle. Free Rad Biol Med. 1991;10:41-7.

8. Hughes HM, George IM, Evans JC, Rowlands CC, Powell GM, Curtis CG. The role of the liver in the production of free radicals during halothane anesthesia in the rat. Quantification of N-tert-butyl-alpha-(4-nitrophenyl)nitrone (PBN)-trapped adducts in bile from halothane as compared with carbon tetrachloride. Biochem J. 1991;277:795-800

9. Jiménez W, Clária J, Arroyo V, Rodés J. Carbon tetrachloride induced cirrhosis in rats: useful tool for investigating the pathogenesis of ascites in chronic liver disease. J Gastroenterol Hepatol. 1992;7:90-7.
10. Knecht KT, DeGray JA, Mason R. Free radical metabolism of halothane in vivo: radical adducts detected in bile. Mol Pharmacol. 1992;41:943-9.

11. Llesuy S, Milei J, Molina H, Boveris A, Milei S. Comparision of lipid peroxidation and myocardial damaged induced by adrimycin and 4-epiadramycin in mice. Tumori. 1985;71:241-9.

12. Maurício T, Marilene P, Miriam S, Moreira AJ, Collado PS, González JG, Marroni NAP. Croton cajucara benth leaves extract scavenges the stable free radical dpph and protects against oxidative stress induced by paraquat. Biol Pharm Bull. 2006;29:161-5.

13. McLain GE, Sipes G, Brown BR Jr. An animal model of halothane hepatotoxicity: roles of enzyme induction and hypoxia. Anesthesiology. 1979;51:321-6.

14. Meneguini R. A toxicidade do oxigênio. Ciência Hoje. 1987;5:57-62.

15. Mikatti NE, Hely TE. Hepatic injury associated with halogenated anaesthetics: cross sensitization and its clinical implications. Eur J Anaesthesiol. 1997;14:7-14.

16. Misra HP, Fridovich I. The role of superoxide anion in the auto-oxidation of epinephrine and a simple assay for superoxide dismutase. J Biol Chem. 1972;247:3170-5.

17. Lind RC, Gandolfi AJ, Hall PD. Covalent binding of oxidative biotransformation intermediates is associated with halothane hepatotoxicity in guinea pigs. Anesthesiology. 1990;73:1208-13.

18. Sipes IG, Brown BR Jr. An animal model of hepatotoxicity associated with halothane anesthesia. Anesthesiology. 1976;45:622-8.

19. Tieppo J, Vercelino R, Dias AS, Marroni C A, Marroni NAP. Ligadura de ducto biliar como modelo de estudo da síndrome hepatopulmonar e estresse oxidativo. Arq Gastroenterol. 2005;42:244-7.

Recebido em 27/3/2006. Aprovado em 21/7/2006. 